\title{
Collisions in primordial star clusters
} Formation pathway for intermediate mass black holes

\author{
B. Reinoso ${ }^{1}$, D. R. G. Schleicher ${ }^{1}$, M. Fellhauer ${ }^{1}$, R. S. Klessen ${ }^{2,3}$, and T. C. N. Boekholt ${ }^{4}$ \\ ${ }^{1}$ Departamento de Astronomía, Facultad Ciencias Físicas y Matemáticas, Universidad de Concepción, Av. Esteban Iturra s/n Barrio \\ Universitario, Casilla 160-C, Concepción, Chile \\ e-mail: breinoso@udec.cl \\ ${ }^{2}$ Universität Heidelberg, Zentrum für Astronomie, Institut für Theoretische Astrophysik, Albert-Ueberle-Str. 2, \\ 69120 Heidelberg, Germany \\ ${ }^{3}$ Universität Heidelberg, Interdisziplinäres Zentrum für Wissenschaftliches Rechnen, Im Neuenheimer Feld 205, \\ 69120 Heidelberg, Germany \\ ${ }^{4}$ CIDMA, Departamento de Física, Universidade de Aveiro, Campus de Santiago, 3810-193 Aveiro, Portugal
}

Received 1 November 2017 / Accepted 17 January 2018

\begin{abstract}
Collisions were suggested to potentially play a role in the formation of massive stars in present day clusters, and have likely been relevant during the formation of massive stars and intermediate mass black holes within the first star clusters. In the early Universe, the first stellar clusters were particularly dense, as fragmentation typically only occurred at densities above $10^{9} \mathrm{~cm}^{-3}$, and the radii of the protostars were enhanced as a result of larger accretion rates, suggesting a potentially more relevant role of stellar collisions. We present here a detailed parameter study to assess how the number of collisions and the mass growth of the most massive object depend on the properties of the cluster. We also characterize the time evolution with three effective parameters: the time when most collisions occur, the duration of the collisions period, and the normalization required to obtain the total number of collisions. We apply our results to typical Population III (Pop. III) clusters of about $1000 M_{\odot}$, finding that a moderate enhancement of the mass of the most massive star by a factor of a few can be expected. For more massive Pop. III clusters as expected in the first atomic cooling halos, we expect a more significant enhancement by a factor of $15-32$. We therefore conclude that collisions in massive Pop. III clusters were likely relevant to form the first intermediate mass black holes.
\end{abstract}

Key words. stars: Population III - dark ages, reionization, first stars - quasars: supermassive black holes

\section{Introduction}

Collisions are often considered to be important during the formation of particularly massive stars, as suggested by Bonnell et al. (1998) and Clarke \& Bonnell (2008). While the formation of low and intermediate mass stars can be readily explained through accretion and infall in a protostellar core (Bodenheimer \& Sweigart 1968; Larson 1969; Shu 1977, see discussion by Palla \& Stahler 1993), 1D models originally failed for stars more massive than $20 M_{\odot}$, which start core hydrogen burning while still accreting, thereby preventing further accretion through ionizing radiation (Yorke \& Sonnhalter 2002). More recent work shows however that in 3D situations, there are always channels through which accretion still occurs, thus not necessarily providing a limiting factor (Keto \& Klaassen 2008; Krumholz et al. 2009; Peters et al. 2010a,b, 2011). A quantitative assessment of the role of collisions in present-day protostellar star clusters has been pursued by Baumgardt \& Klessen (2011), finding that between $0.1 \%$ and $1 \%$ of the protostars participate in such collisions within typical clusters, potentially providing a relevant enhancement for sufficiently large numbers of stars. Similar investigations have been pursued by Moeckel \& Clarke (2011), Oh \& Kroupa (2012a), and Fujii \& Portegies Zwart (2013).

In the early Universe, the conditions are potentially even more favorable for the formation of massive objects via collisional processes. In the primordial gas, the cooling is less efficient and predominantly driven by small fractions of molecular hydrogen (see, e.g., Omukai et al. 2005). As shown via numerical simulations, the typical densities where fragmentation occurs are on the order $10^{9} \mathrm{~cm}^{-3}$ or higher (e.g., Clark et al. 2011a,b; Greif et al. 2011, 2012; Smith et al. 2011, 2012; Latif et al. 2013b), leading to the formation of dense clusters with radii of $0.1 \mathrm{pc}$ or even smaller. Trace amounts of dust grains may even trigger fragmentation at still higher densities (e.g., Schleicher et al. 2003, 2006, 2012; Omukai et al. 2008; Klessen et al. 2012; Dopcke et al. 2011, 2013; Bovino et al. 2016; Latif et al. 2016), providing ideal conditions for the formation of very dense clusters.

In addition to the compactness of the cluster, the protostellar radii are also enhanced by the more rapid accretion expected in primordial or low-metallicity gas, thereby increasing the overall cross section for collisions. Large protostellar radii of up to $300 R_{\odot}$ were calculated in stellar evolution models by Stahler et al. (1986) or Omukai \& Palla (2001, 2003). It has been shown that variable protostellar accretion rates can further enhance these radii (Smith et al. 2012). A strong increase of the radii also seems possible in the presence of particularly high accretion rates of $\sim 0.1 M_{\odot} \mathrm{yr}^{-1}$ (Hosokawa et al. 2012, 2013; Schleicher et al. 2013; Haemmerlé et al. 2018; Woods et al. 2017), implying 
$\sim 500 R_{\odot}$ for a $10 M_{\odot}$ star and potentially more than $1000 R_{\odot}$ for a $100 M_{\odot}$ star.

It is thus conceivable that collisions may play some role in typical Population III (Pop. III) clusters as expected to form in so-called minihalos with about $10^{6} M_{\odot}$ and in the more massive atomic cooling halos with masses on the order $10^{8} M_{\odot}$. These more massive halos are frequently considered as the birth places of intermediate mass black hole seeds. Collisional processes were indeed mentioned as an important pathway in the seminal paper by Rees (1984), and subsequently taken into account for instance in semianalytical models by Devecchi et al. (2010, 2012) and Lupi et al. (2014). In $N$-body simulations employing cosmological initial conditions, Katz et al. (2015) and Sakurai et al. (2017) have shown that black holes with masses of $\sim 10^{3} M_{\odot}$ can be formed.

We present a systematic investigation on how the formation of very massive objects depends on the properties of the cluster. Our numerical setup and the initial conditions are described in Sect. 2, and our results are described in Sect. 3, including the time evolution of a typical cluster, the number of collisions and mass of the resulting object found under different conditions, along with the time required to achieve such an enhancement. In Sect. 4 , the results are applied to primordial clusters, both in the context of minihalos and the larger atomic cooling halos. A final summary and discussion is given in Sect. 5.

\section{Simulation setup}

We present a set of $N$-body simulations of stellar collisions in compact star clusters. We have not included the effect of a gaseous potential or modeled the effect of the gas in explicit terms. To zero order, if the latter is dominating the potential, it will primarily increase the velocity dispersion and thereby decrease the crossing time of the cluster, allowing for a larger number of crossing times in a given physical time. In addition, there can be other gas-related effects that may enhance the number of collisions, which we discuss in Sect. 3.5. Our calculations thus provide a conservative lower limit on the number of collisions. To perform the calculations we used a modified version of NBODY6 ${ }^{1}$ (Aarseth 2000) to treat collisions, in which we switch off the stellar evolution package and instead explicitly specify the stellar radii to perform a parameter study. The NBODY6 program is a fourth order Hermite integrator that includes a spatial hierarchy to speed up the calculations known as the Ahmad-Cohen scheme (Ahmad \& Cohen 1973). This program also includes routines to treat tidal circularization, which is believed to be the main mechanism from which binaries are formed in star clusters. Another important routine included is the Kustaanheimo-Stiefel regularization (Kustaanheimo \& Stiefel 1965), an algorithm to treat binaries and close two body encounters more accurately and faster.

\subsection{Star clusters}

We investigated how the number of collisions and the mass of the final object depends on the number of stars $N$ and the radii of the stars $R_{\text {star }}$. We modeled a compact cluster in virial equilibrium consisting of equal mass stars with a total stellar mass of $M_{\text {cluster }}=10^{4} M_{\odot}$ using a Plummer distribution for the stars (Plummer 1911) with a Plummer radius of $R_{p l}=0.077 \mathrm{pc}$, implying a half-mass radius of $R_{h}=0.1 \mathrm{pc}$. With this configuration

\footnotetext{
1 Webpage NBODY6: https://www.ast.cam.ac.uk/ sverre/ web/pages/nbody.htm
}

the crossing time of the cluster is $0.022 \mathrm{Myr}$. We varied the number of stars $N=100,500,1000,5000$ and kept the mass of the cluster constant, therefore the initial masses of the stars $M_{\text {ini }}$ depend on $N$ as $M_{\text {ini }}=M_{\text {cluster }} / N$. For a fixed number of stars $N$ we varied the stellar radii $R_{\text {star }}=20,50,100,200,500,1000$, and $5000 R_{\odot}$.

\subsection{Stellar collisions}

The standard version of NBODY6 includes a routine to treat stellar collisions and can be used only when the stellar evolution option is activated. The stellar evolution package included in the code is useful for metallicities ranging from $Z=0.0001$ up to $Z=0.03$ (Hurley et al. 2000). In this investigation, we are primarily interested in the application to extremely metal poor conditions that have metallicities $Z \leq 10^{-6}$. Instead of pursuing detailed stellar evolution calculations, our goal here is to determine the regime where the collisions are potentially relevant. To treat the collisions, we therefore switch off the stellar evolution routine in NBODY6, and model the collisions as follows:

A collision occurs when the separation $d$ between two stars is smaller than the sum of the radii of the stars $\left(d \leq R_{1}+R_{2}\right)$. When this condition is satisfied, we replaced the two colliding stars by a new star, and the mass of the new star $M_{\text {new }}$ is simply the sum of the masses of the two colliding stars $M_{\text {new }}=M_{1}+M_{2}$. The radius of the new star $R_{\text {new }}$ is calculated with Eq. (1) using the condition that the new star should have the same density as the colliding stars, that is,

$R_{\text {new }}=R_{1}\left(\frac{M_{1}+M_{2}}{M_{1}}\right)^{1 / 3}$.

Effectively, this assumes that the collision product quickly settles into a new equilibrium configuration in which the density corresponds to that of an unperturbed star of the same mass. This is consistent with detailed stellar evolution calculations, for example, by Hosokawa et al. (2012) or Haemmerlé et al. (2016). Nevertheless, we emphasize that we do not aim to follow the detailed stellar evolution.

\section{Results}

In the following, we describe the main results of our calculations. This includes the description of the time evolution in a typical cluster, the number of collisions that occur for various cluster parameters assuming a sufficient time of integration, a description of the time evolution and its parametrization, a discussion of ejections, and a discussion on uncertainties and neglected processes.

\subsection{Time evolution in a typical cluster}

The time evolution in two typical clusters is shown in Figs. 1 and 2, the first shows a cluster with 1000 stars, each of which has a radius of $500 R_{\odot}$. The second is a cluster with 5000 stars, each of which has the same stellar radius $\left(500 R_{\odot}\right)$. For both clusters, we show the fraction of stellar collisions (relative to the total initial number of stars), the logarithm of the Lagrangian radii corresponding to $10 \%, 50 \%$, and $90 \%$ of the mass, and the mass of the most massive object normalized through the initial mass of the stars.

In Fig. 1, the maximum of collisions occurs clearly between 30 and 50 crossing times, where the Lagrangian radius corresponding to $10 \%$ of the enclosed mass also drops considerably, 


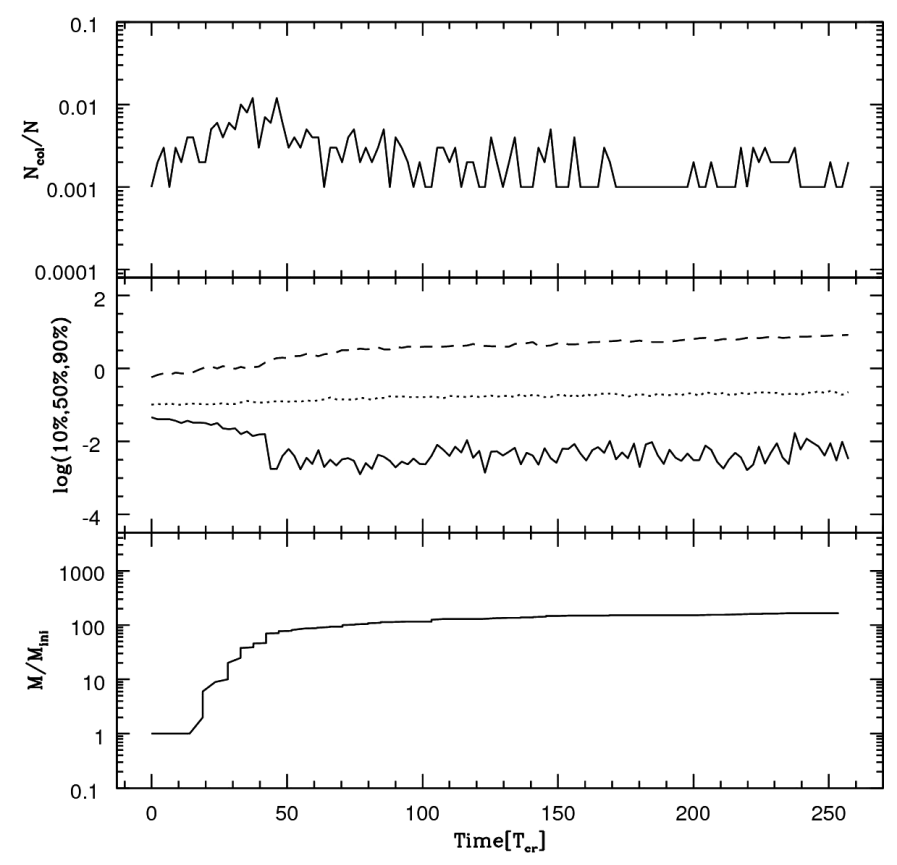

Fig. 1. Time evolution of a cluster with $N=1000$ stars. Each of these stars has an initial radii of $R_{\text {star }}=500 R_{\odot}$. Top panel: number of collisions divided by number of stars as a function of time is shown. Middle panel: logarithm of the Lagrangian radii corresponding to $10 \%, 50 \%$, and $90 \%$ of the enclosed mass as a function of time is shown. Bottom panel: mass of the most massive object divided by the initial stellar mass as a function of time is shown. In all panels, the time is normalized by the crossing time of the cluster.

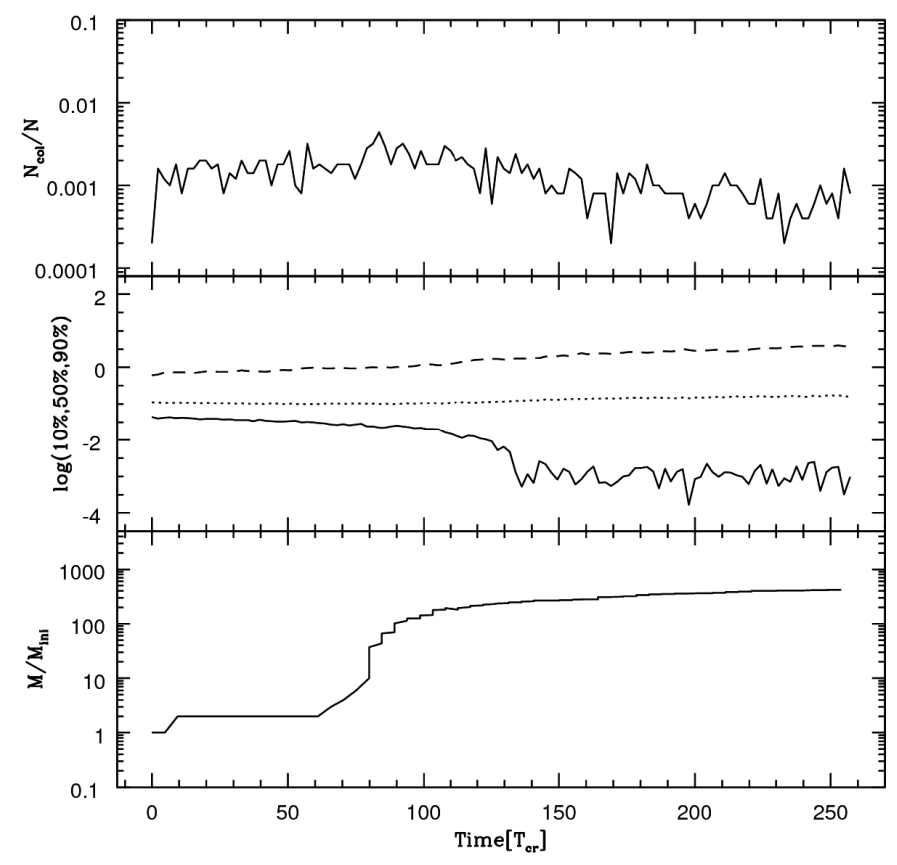

Fig. 2. Time evolution of a cluster with $N=5000$ stars. Each of these stars has an initial radii of $R_{\text {star }}=500 R_{\odot}$. Same panels as in Fig. 1 .

revealing that a massive central object has already accreted $10 \%$ of the mass at this time. Most of the collisions occur within the half-mass radius of the cluster and more massive stars are formed very close to the center of the cluster where they collide to form a single very massive object. The peak fraction of collisions reaches about $1 \%$, and the mass of the central object increases
Table 1. Parameters of Eqs. (2) and (3).

\begin{tabular}{lcccc}
\hline \hline$N$ & $B$ & $C$ & $D$ & $E$ \\
\hline 100 & $0.45 \pm 0.02$ & $-2.25 \pm 0.06$ & $0.40 \pm 0.04$ & $-0.16 \pm 0.09$ \\
500 & $0.49 \pm 0.02$ & $-2.27 \pm 0.06$ & $0.53 \pm 0.04$ & $0.22 \pm 0.09$ \\
1000 & $0.51 \pm 0.04$ & $-2.29 \pm 0.09$ & $0.57 \pm 0.06$ & $0.44 \pm 0.14$ \\
5000 & $0.50 \pm 0.03$ & $-2.16 \pm 0.07$ & $0.52 \pm 0.05$ & $1.28 \pm 0.12$ \\
\hline
\end{tabular}

Notes. Parameters from the fit to Eqs. (2) (Cols. 2 and 3) and (3) (Cols. 4 and 5) to estimate the total fraction of collisions in a cluster depending on the initial radii of the stars $R_{\text {star }}$ and the initial number $N$ of stars (Eq. (2)). Parameters $D$ and $E$ are used to estimate the mass of the most massive object divided by its initial mass $\left(M_{\max } / M_{\text {ini }}\right)$ at the end of the runaway growth, depending on the initial radii $R_{\text {star }}$ of the stars and the initial number $N$ of stars (Eq. (3)).

by a corresponding factor of about 100 because of a similar number of stellar collisions. The $50 \%$ and $90 \%$ Lagrangian radius shows sings of expansion, as the cluster dissolves due to ejections.

The behavior in Fig. 2 is very similar. The main difference is that the time over which the collisions occur is more spread out, ranging from 10 up to $130-150$ crossing times. While the fraction of collisions is approximately constant during that period, with values are around $0.1 \%$, the runaway growth of the most massive object begins at around 80 crossing times, suggesting that before that stage several more massive stars had formed and subsequently merged. Once the mass of the most massive object has increased by about a factor of 500, thus corresponding to about $10 \%$ of the total mass, the corresponding Lagrangian radius decreases as seen in Fig. 1. The overall evolution in both cases is thus very similar.

\subsection{Number of collisions for various cluster parameters}

Our central goal is to determine how the number of collisions and the growth of the most massive object depends on the properties of the cluster. For this purpose, we initially assume that enough time is available until all collisions have occurred, while investigating more details of the time evolution in the next subsection.

Our main results are given in Fig. 3, showing the fraction of collisions as a function of stellar radius and for various numbers of stars. The data shown here correspond to an average of more than simulations for each individual configuration to improve the statistical reliability of the results. We find that the total fraction of collisions depends very weakly on the number of stars, showing an increase by at most a factor of 2 when going from 100 to 5000 stars. This very minor increase may result from the fact that a larger (but still small) fraction of the effective area is filled with stars. We do not in principle expect this to depend strongly on the IMF, but it remains one of the uncertainties to be explored in future studies. The dependence on the stellar radii, on the other hand, is very clear and corresponds to a power law with slope of about 0.5 (see second column in Table 1).

The mass of the most massive object normalized by the initial mass of the stars shows a clearer dependence both on the number of stars and on the radii of the star. This can be understood from the fact that the fraction of collisions is roughly independent of the number of stars. If most collisions occur with the most massive object (as we have checked), the fraction of the mass going into the most massive object is approximately constant, and thus normalizing this mass by the initial mass of the 

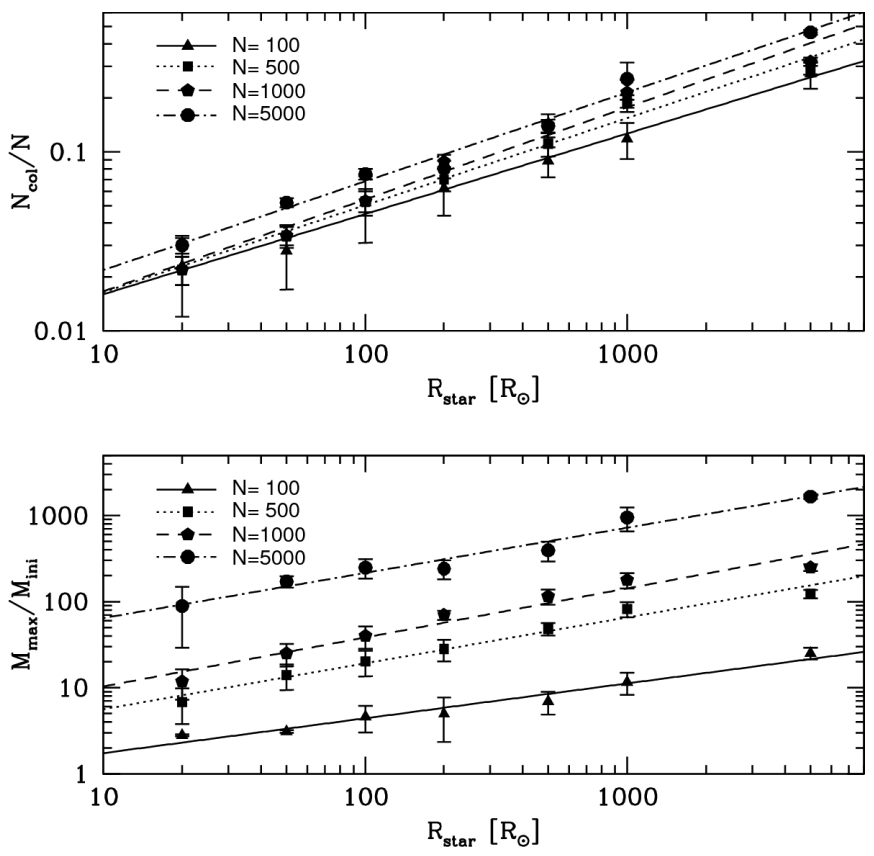

Fig. 3. Top panel: fraction of collisions $\left(N_{\text {col }} / N\right)$ as a function of the initial stellar radius and the number of stars. We also show the best linear fits from Eq. (2) and the parameters for these fits in Table 1. Bottom panel: final mass over the initial mass $\left(M_{\max } / M_{\mathrm{ini}}\right)$ for the most massive star at the end of the runaway growth as function of the initial stellar radius and the initial number of stars. We also show the best linear fits from Eq. (3) and the parameters for these fits in Table 1.

stars leads to a dependence on the number of stars, as the cluster mass is fixed. As a function of stellar radius, this quantity indeed shows a power-law behavior with a slope of about $1 / 3$. We fit the data using an implementation of the nonlinear least-squares Marquardt-Levenberg algorithm in gnuplot with the functions

$\log \left(\frac{N_{\text {col }}}{N}\right)=B \log \left(R_{\text {star }}\right)+C$,

$\log \left(\frac{M_{\text {max }}}{M_{\text {ini }}}\right)=D \log \left(R_{\text {star }}\right)+$ E.

The parameters of the fit are shown in Table 1. We found that $E, C$ does not depend on $N$, however even if these parameters are constant, the total number of collisions $N_{\text {col }}$ still depends on $N$ as described in Eq. (2). We also found that $D$ is constant but $E$ depends on $N$ as follows:

$E=0.84 \pm 0.13 \log (N)-1.96 \pm 0.39$.

\subsection{Description of the time evolution}

For many practical applications, it is necessary to have more information on the time evolution of the collisions. While a detailed description of every run is clearly unfeasible, our goal is to extract three main parameters that contain the most essential information as follows: the time delay when most of the collisions occur, the duration of this period, and the total number of collisions. For this purpose, a Gaussian fit is applied to the number of collisions over time. The parameter $t_{\text {delay }}$ describes the time when most collisions occur, $t_{\text {duration }}$ approximates the duration of the collision period. The fit is given as

$N_{\text {col }}=A \exp \left(-\frac{\left(t-t_{\text {delay }}\right)^{2}}{2 t_{\text {duration }}^{2}}\right)$.

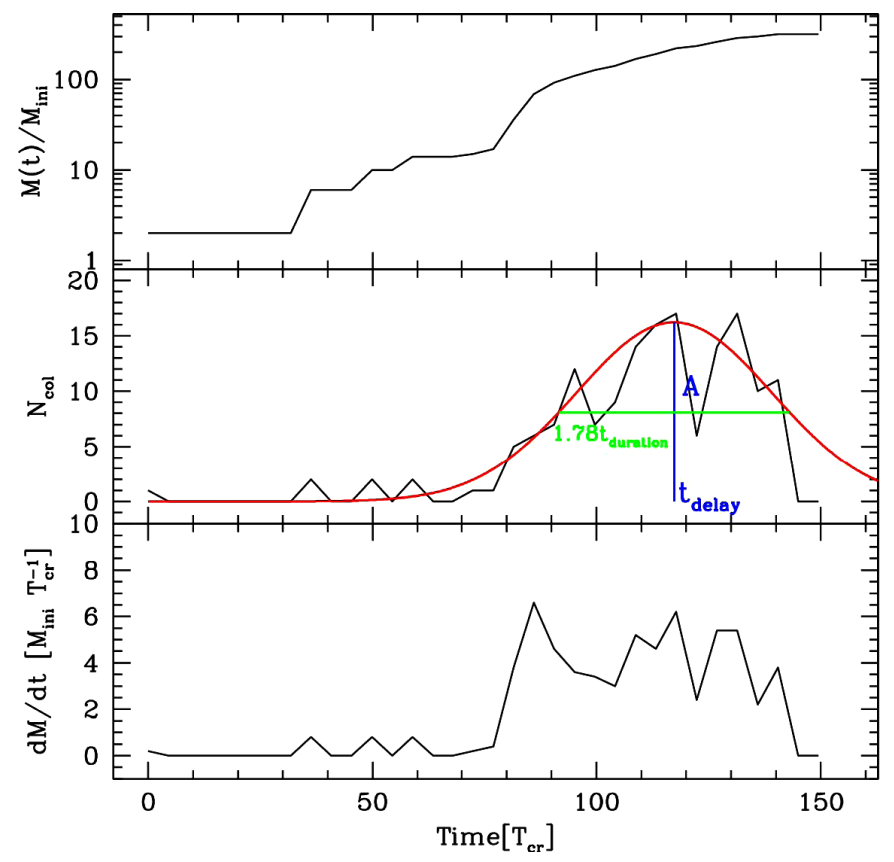

Fig. 4. Top panel: mass evolution of the runaway $\operatorname{star}\left(M_{\max }(t) / M_{\mathrm{ini}}\right)$ in a cluster with $N=5000$ stars. Each of these stars has an initial mass of $M_{\text {star }}=2 M_{\odot}$ and an initial radius of $R_{\text {star }}=200 R_{\odot}$. Middle panel: the number of collisions in bins of 5 crossing times $\left(N_{\mathrm{col}}\right)$ as function of the crossing time of the cluster is shown. The red line is the best Gaussian fit. Bottom panel: the mass growth rate $\mathrm{d} M / \mathrm{d} t$ in $M_{\mathrm{ini}} T_{\mathrm{cr}}^{-1}$ calculated in bins of 5 crossing times is shown.

The normalization $A$ is adopted to ensure that the total number of collisions is correctly reproduced.

An example for such a fit is given in Fig. 4, showing the number of collisions in a cluster with 5000 stars and a stellar radius of $500 R_{\odot}$ as a function of time. The results shown in Fig. 4 are calculated in bins of five crossing times as there are few collisions per crossing time and we aim for a general description of the effect of collisions rather than a more individual description that depends more on statistical variations. Nevertheless a smaller or larger bin size does not change our results as we have checked. While the time evolution of the collisions does not precisely follow a Gaussian distribution, we find that the time when most collisions occur and the duration of the collisions is described very well by the Gaussian fit. For comparison, we also show the time evolution of the mass of the most massive object divided by the initial mass as a function of time. The steepest growth in mass occurs around 80 crossing times owing to a collision with a $40 M_{\odot}$ star formed also through stellar collisions; however, the number of collisions peaks around 120 crossing times and this agrees with a second peak in the mass enhancement, which is produced at this stage by several mergers with smaller stars. The width of the Gaussian fit matches about the time when the central object stops growing in mass. Given the overall uncertainties in the problem considered, the latter provides a reasonable description of the most relevant information.

The resulting fit parameters are given in Fig. 5 as functions of stellar radius and for different numbers of stars. The parameter $A$ increases by a factor of 4 between 20 and $5000 R_{\odot}$ for a cluster with 100 stars, while the increase is more enhanced, corresponding to about a factor of 20 , for a cluster with 1000 stars. The increase of the parameter $A$ is more moderate than naively expected from the behavior of the total fraction of collisions, which is partly due to how the duration of the collision 


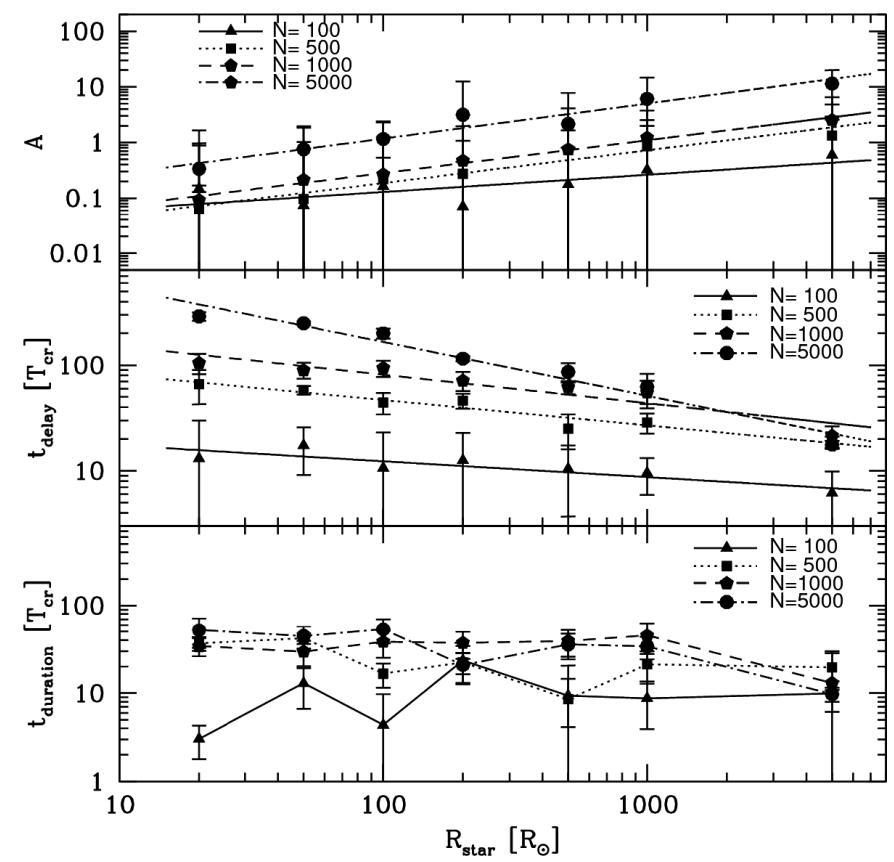

Fig. 5. Parameters of the Gaussian fit $\mathrm{A}, t_{\text {delay }}$, and $t_{\text {duration }}$ as functions of the initial stellar radii $\left(R_{\text {star }}\right)$ and initial number of stars $N$.

period changes with the number of stars. In particular, the evolution of the $t_{\text {duration }}$ parameter as a function of stellar radius is relatively flat (slightly decreasing), and only weakly dependent on the number of stars. However, a decreasing value of $t_{\text {delay }}$, which quadratically enters the exponential, can overcompensate for the behavior of $A$. The parameter $t_{\text {delay }}$ describing the time when most of the collisions occur, on the other hand, decreases with stellar radius and increases with the number of stars. This reflects in particular the increase of the relaxation time in the cluster with the number of stars and the increased probability of collisions with increasing stellar radius. We fit a linear function to the quantities $\log (A)$ and $\log \left(t_{\text {delay }}\right)$, where $t_{\text {delay }}$ is expressed in units of the crossing time $T_{c r}$ of the cluster, depending on the logarithm of the stellar radii as follows:

$\log (A)=\alpha \log \left(R_{\text {star }}\right)+\beta$

$\log \left(t_{\text {delay }}\right)=\gamma \log \left(R_{\text {star }}\right)+\delta$.

The parameters $\alpha, \beta, \gamma$, and $\delta$ are shown as functions of the number of stars $N$ in Table 2 and the fitting lines are shown in Fig. 5.

There is no clear dependence of the duration of the runaway growth on stellar radii (see bottom panel of Fig. 5). For that reason we explore the dependence of this parameter on the number of stars $N$. We find an exponential dependence for the duration of the runaway growth as a function of the number of stars as shown in Fig. 6 . The relation between $t_{\text {duration }}$ and $N$ is described as

$\log \left(t_{\text {duration }}\right)=0.34 \pm 0.08 \log N+0.34 \pm 0.24$.

As we find no clear relation between $t_{\text {duration }}$ and $R_{\text {star }}$ we calculate this parameter only as a function of $N$ from Eq. (8) and assume that $t_{\text {duration }}$ is constant for all stellar radii. On the other hand, we find clear relations for $A\left(R_{\text {star }}\right)$ and $t_{\text {delay }}\left(R_{\text {star }}\right)$, however, these relations depend also on $N$ (see Fig. 5). In order to get an approximate value for the number of collisions experienced by the runaway star in a star cluster consisting of $N$ particles with a
Table 2. Parameters of Eqs. (6) and (7).

\begin{tabular}{lcccc}
\hline \hline$N$ & $\alpha$ & $\beta$ & $\gamma$ & $\delta$ \\
\hline 100 & $0.31 \pm 0.12$ & $-1.51 \pm 0.29$ & $-0.15 \pm 0.04$ & $1.39 \pm 0.09$ \\
500 & $0.59 \pm 0.06$ & $-1.91 \pm 0.16$ & $-0.24 \pm 0.03$ & $2.15 \pm 0.07$ \\
1000 & $0.63 \pm 0.07$ & $-1.19 \pm 0.19$ & $-0.27 \pm 0.05$ & $2.45 \pm 0.11$ \\
5000 & $0.59 \pm 0.03$ & $-1.73 \pm 0.07$ & $-0.51 \pm 0.05$ & $3.24 \pm 0.12$
\end{tabular}

Notes. Parameters from the fit to Eqs. (6) and (7) to estimate the normalization parameter $A$ used to get the total number of collisions with the central runaway star in a cluster depending on the initial stellar radii $R_{\text {star }}$ (Eq. (6)). These parameters are also used to estimate the parameter $t_{\text {delay }}$, which is related to the time when the rate of collisions with the central runaway star is maximum; this also depends on the initial radii $R_{\text {star }}$ of the stars (Eq. (3)).

radii of $R_{\text {star }}$, we need to take into account the dependence of $A$ and $t_{\text {delay }}$ on $N$.

Equations (6) and (7) show the dependence of $A$ and $t_{\text {delay }}$ on $R_{\text {star }}$. The slope of these relations depends on the number of stars $N$ as described in Table 2. In order to properly account for the dependence on $N$ when calculating the number of collisions experienced by the runaway star, we fit a line for the parameters $\alpha, \beta, \gamma$, and $\delta$ (related to the estimation of $A$ and $t_{\text {delay }}$ ) as functions of $N$. The relations we found are described as

$\alpha=0.16 \pm 0.09 \log N+0.06 \pm 0.27$
$\beta=-0.05 \pm 0.31 \log N-1.43 \pm 0.90$
$\gamma=-0.21 \pm 0.05 \log N+0.30 \pm 0.13$
$\delta=1.09 \pm 0.01 \log N-0.79 \pm 0.04$

If we then combine Eqs. (6), (7), and (9)-(12), we find $A$ and $t_{\text {delay }}$ as functions of $N$ and $R_{\text {star }}$ as described in Eqs. (13) and (14), that is,

$$
\begin{aligned}
\log (A)=[ & \left.0.16 \log \left(R_{\text {star }}\right)-0.05\right] \log (N) \\
+ & 0.06 \log \left(R_{\text {star }}\right)-1.43 \\
\log \left(t_{\text {delay }}\right)= & {\left[-0.21 \log \left(R_{\text {star }}\right)+1.09\right] \log (N) } \\
& +0.30 \log \left(R_{\text {star }}\right)-0.79 .
\end{aligned}
$$

Finally, the number of collisions experienced by the runaway star in a cluster of $N$ stars with radii of $R_{\text {star }}$ between times $t_{1}$ and $t_{2}$ is given by

$N_{\text {col }}=A \int_{t_{1}}^{t_{2}} \exp \left\{\frac{-\left(t-t_{\text {delay }}\right)^{2}}{2 t_{\text {duration }}^{2}}\right\} \mathrm{d} t$,

where $A$ is calculated from Eq. (13), $t_{\text {delay }}$ is calculated from Eq. (14), and $t_{\text {duration }}$ is calculated from Eq. (8). Time is expressed in units of the crossing time of the cluster.

We solve Eq. (15) using the relations found for $A, t_{\text {delay }}$, and $t_{\text {duraion }}$ as functions of $N$ and $R_{\text {star }}$ for $50 \leq N \leq 10000$ and $10 \leq R_{\text {star }} \leq 2000$. We show the expected number of collisions with the central object after $1 \mathrm{Myr}$ and $10 \mathrm{Myr}$ in Fig. 7. If collisions need to take place within $10^{6} \mathrm{yr}$, owing to the lifetime of the most massive star, the strongest collisional contributions may occur for clusters with about 1000 stars, as otherwise the characteristic timescale for the collisions to set in becomes too long. In case of stellar radii of $100 R_{\odot}$, the enhancement by collisions corresponds to a factor of a few, which increases strongly for larger 


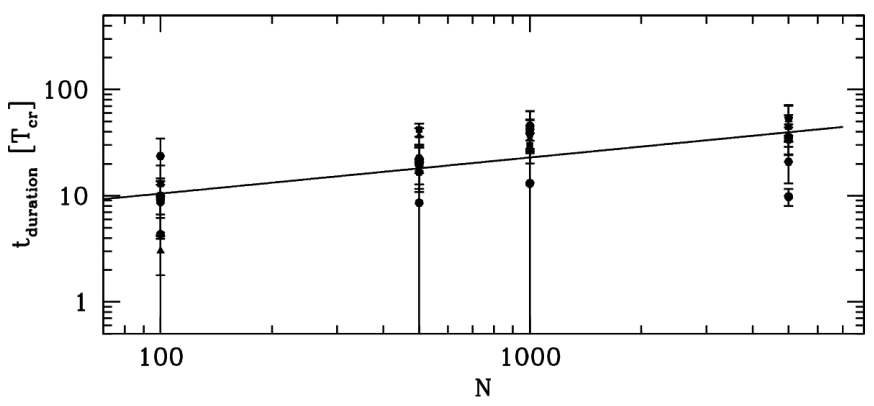

Fig. 6. Parameter $t_{\text {duration }}$ of the Gaussian fit as function of the number of stars $N$. We assumed that $t_{\text {duration }}$ only depends on $N$ and the fit is described in Eq. (8).

radii. In case the time available to form very massive objects is 10 million years, then the number of collisions increases as a function of $N$ until a value of 5000 or higher, depending on the stellar radii. In such cases, relevant enhancements can be found even for stellar radii of $20 R_{\odot}$, and potentially much larger for larger radii. This case is therefore the most promising case for the formation of very massive objects. It basically requires stars with less than $20 M_{\odot}$, as their lifetime is then longer than $10 \mathrm{Myr}$ (Schaerer 2002). Our simulations show that in stellar systems containing equal mass stars with masses $M \leq 20 M_{\odot}$, the expected enhancement factor within $10 \mathrm{Myr}$ ranges from 11 to 50 times the initial stellar mass $M_{\text {ini }}$, considering an initial stellar radius of $100 R_{\odot}$ and depending on the number of stars.

In summary, we provided a fit for $N_{\text {col }}$ that depends on the typical duration of the collision phase $t_{\text {duration }}$, the delay time until collisions occur $t_{\text {delay }}$, and the overall normalization $A$. We found that $A$ and $t_{\text {duration }}$ predominantly depend on the number of stars $N$, while $t_{\text {delay }}$ depends both on $N$ and $R_{\text {star }}$. We provided fits for the functional dependence of these parameters, and also demonstrate how the number of collisions that has occurred after 1 and 10 million years depends both on $N$ and $R_{\text {star }}$. We found that, as the delay time increases with $N$, the number of collisions within $10^{6} \mathrm{yr}$ does not strongly increase with $N$ at fixed $R_{\text {star }}$, but it does increase when considering a time of $10 \mathrm{Myr}$.

\subsection{Ejections from the cluster}

We also investigate the number of ejection events, which are shown in Fig. 8 both as a function of stellar radius and as a function of the number of stars. A star is considered unbound once its distance is $20 R_{\mathrm{vir}}$. For all the clusters modeled in this work, the virial radius is $R_{\mathrm{vir}}=0.14 \mathrm{pc}$; thus a star has escaped the cluster when it is at $2.8 \mathrm{pc}$ away from the cluster center. The fraction of escaped stars seems to vary from a few up to about $20 \%$. While there is a large scatter for a given stellar radius, the escape fraction seems independent of that quantity, while it appears to slightly decrease with the number of stars. This may particularly reflect that clusters with low numbers of stars of order 100 dissolve more easily and do not provide a well-sampled statistical distribution. We further checked that the velocity of the escaping stars is independent of stellar radius and number of stars, and corresponds to the expected escape velocity given the mass and radius of the cluster.

While stellar ejections have not yet been systematically explored in Pop. III clusters, their potential role has been examined in various contexts. For instance, Pflamm-Altenburg \& Kroupa (2010) investigated the combined effect of massive

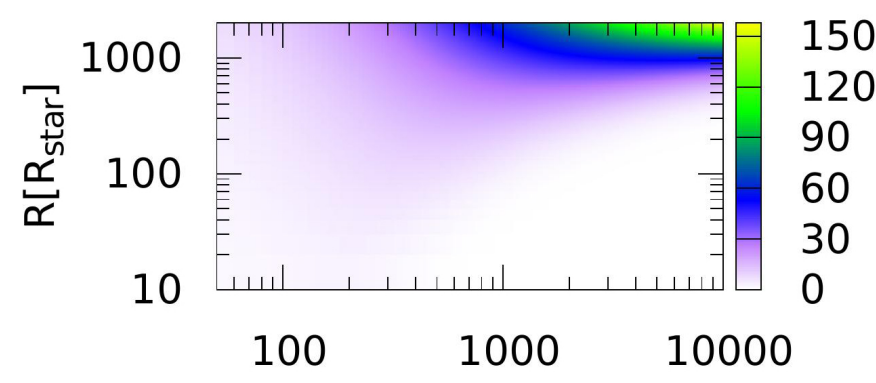

$\mathrm{N}$

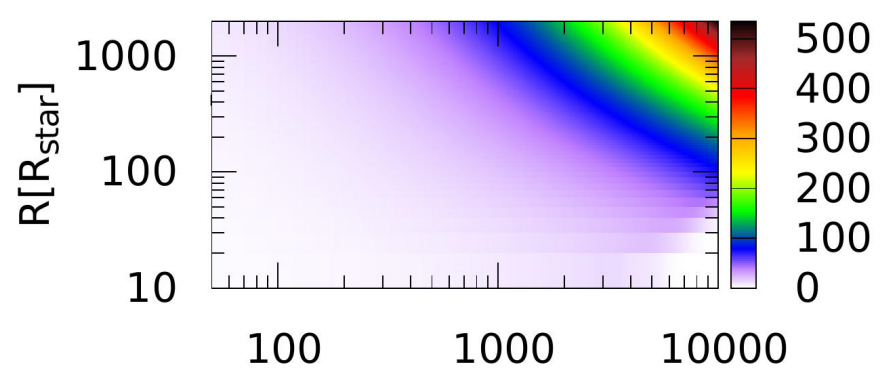

$\mathrm{N}$

Fig. 7. Total number of collisions calculated via Eq. (15) (shown through color) with the central object after $1 \mathrm{Myr}$ (top panel) and after $10 \mathrm{Myr}$ (bottom panel) for our cluster model depending on the number of stars and their initial radii.

binary ejections from star clusters, including a second acceleration of a massive star during a subsequent supernova. The latter may potentially help to understand the observed fraction of isolated O-star formation candidates. Oh \& Kroupa (2012b) explored how the ejection of massive stars affects the relation between maximum stellar mass and cluster mass. They found that lower mass clusters do not shoot out their heaviest star, while this may occur in more massive mass clusters. These results have been further refined by Oh et al. (2015) and Oh \& Kroupa (2016), showing that star clusters of $400 M_{\odot}$ are likely the dominant sources for $\mathrm{O}$ stars.

\subsection{Uncertainties and neglected processes}

In this investigation, we have not included the effect of a gaseous potential or modeled the effect of the gas in explicit terms. To zero order, if the latter dominates the potential, it will primarily increase the velocity dispersion and thereby decrease the crossing time of the cluster, allowing for a larger number of crossing times in a given physical time. In addition, the gas may provide dynamical friction, which can potentially favor the probability of collisions. The effects of dark matter are also neglected given that at the point when the star clusters are formed, the gravitational potential is dominated by the baryons (Abel et al. 2002; Bromm \& Loeb 2003).

Related to this, the accretion onto the protostars was not explicitly considered, and their masses were taken to be constant. In cases in which accretion contributes to the mass growth, this may again favor collisions and induce mergers of binaries that otherwise would be stable.

A further simplifying assumption employed here was that we did not consider a full initial mass function (IMF), but assumed initially uniform stellar masses. The latter has the 

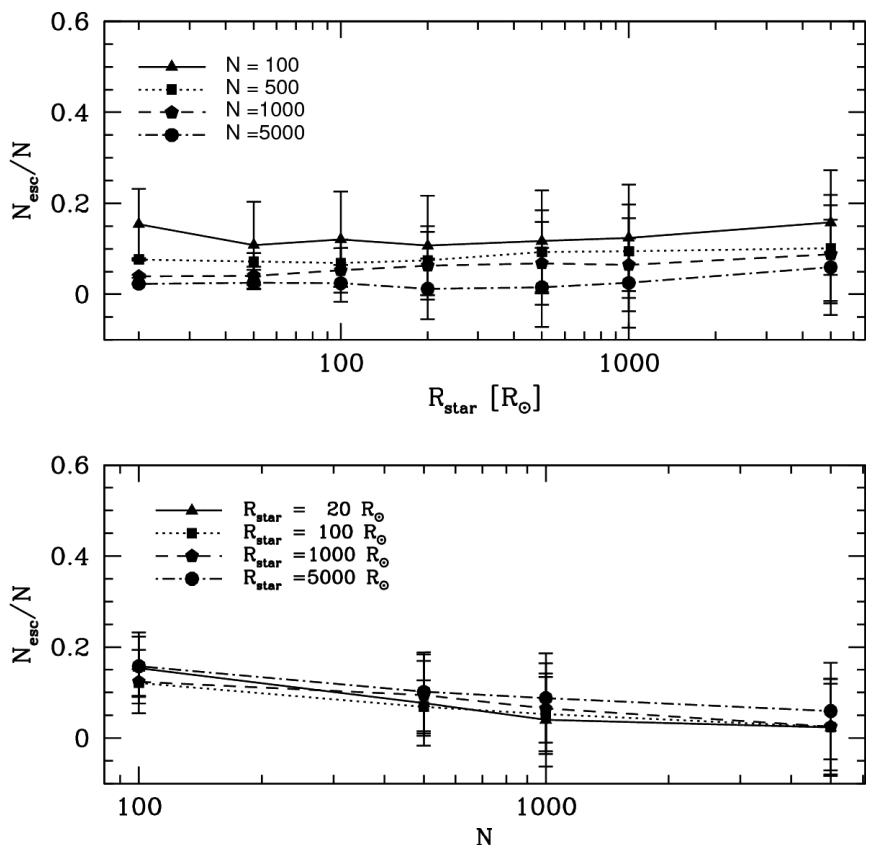

Fig. 8. Top panel: fraction of ejections (number of ejected stars divided by the initial number of stars) as a function of the initial stellar radius. Bottom panel: fraction of ejections (number of ejected stars divided by the initial number of stars) as a function of the initial number of stars.

effect to suppress three-body ejections, which may otherwise be more frequent and preferably eject low mass stars. In the presence of a logarithmically flat IMF, as it is often assumed in the context of primordial star formation (Greif et al. 2012; Hartwig et al. 2016), we do not expect this to be a major problem, although it can possible reduce the mass of the most massive object by a certain degree. We note also that the simulations by Sakurai et al. (2017) have included a full IMF, which however has not prevented the formation of a $1000 M_{\odot}$ object.

In cases in which a full IMF is considered, the next step is then clearly to take the dependence of the mass on stellar radius and the evolution of the stellar radii over time into account. In this sense, our current simulations represent an effective model in which an average stellar radius is adopted over the period of time considered. This effective stellar radius should correspond to the typical stellar radius at the time when the majority of collisions is expected to occur. In addition, also the so-called hit-and-stick assumption employed here is valid for

$\sigma_{\text {stars }}^{2}<G \frac{M_{\text {star }}}{R_{\text {star }}}$.

The velocity dispersion of the stars in all our simulations is $12.39 \mathrm{~km} \mathrm{~s}^{-1}$, thus the hit-and-stick approximation should be valid for all our models except when $R_{\text {star }}=5000 R_{\odot}$. However this approximation still needs to be tested in future simulations, both to investigate how much material needs to stick for efficient growth still to occur, but also specific simulations exploring stellar collisions and merger processes would be valuable for a better understanding of possible limitations.

While clearly the processes mentioned here deserve further investigations, we show below that the results obtained above clearly suggest the potential relevance of collisions in primordial clusters.

\section{Implications for primordial clusters}

In the following, we explore the implications of our results with respect to primordial star clusters. We distinguish here in particular the case of standard Pop. III clusters as expected in a typical minihalo with about $10^{6} M_{\odot}$, and a more massive atomic cooling halo with about $10^{8} M_{\odot}$.

\subsection{Standard Pop. III clusters (minihalos)}

For a typical Pop. III star cluster, we assumed a mass of $1000 M_{\odot}$, which is consistent with a baryon fraction of about $10 \%$ and a star formation efficiency of order $1 \%$ in a $10^{6} M_{\odot}$ minihalo. We adopted a radius of the cluster of order $0.1 \mathrm{pc}$, which is consistent with results from simulations and semianalytic models (Clark et al. 2011a,b; Greif et al. 2011, 2012; Latif et al. 2013a; Latif \& Schleicher 2015). We take a stellar radius of $100 R_{\odot}$, which is characteristic for primordial protostars with accretion rates on the order $10^{-3} M_{\odot} \mathrm{yr}^{-1}$ (Hosokawa et al. 2012). The crossing time of the cluster then corresponds to 0.071 Myr. The number of stars that can be expected in such a cluster is uncertain, but we adopted an estimate of about 100 .

Using the relations we found, we expect a total of about four collisions to occur within 1 million years. This is unchanged even if we assume a lifetime of $10 \mathrm{Myr}$, as the runaway growth occurs within 1 Myr. We expect the lifetime of a massive primordial star to be in between this range, depending on precise mass, amount of rotation, and the effects the collisions may have on the stellar evolution (e.g., Maeder \& Meynet 2012). We therefore find that a moderate enhancement can be achieved within a normal cluster.

We note that the values given here are the expected mean number of collisions. Individual clusters can deviate from these both toward lower and higher fractions of collisions, including potentially clusters with zero collisions. Especially for typical Pop. III clusters in which the number of collisions is low, the uncertainty in the collision fraction can be expected to be comparable to the mean value.

\subsection{Massive primordial clusters (atomic cooling halos)}

As a next step, we address now the potential impact of collisions in a more massive atomic cooling halo. Under the right conditions, in particular if the cooling on larger scales is regulated by atomic hydrogen (e.g., Latif et al. 2014), a rather massive cluster of $10^{4} M_{\odot}$ can form, which is exposed to larger accretion rates on the order $10^{-1} M_{\odot} \mathrm{yr}^{-1}$. We assume that the cluster consists of an initial amount of 1000 stars, and that the stellar radii are somewhat enhanced compared to the standard Pop. III cluster due to the higher accretion, with a typical value of about $300 R_{\odot}$. The crossing time in the cluster is then 0.023 Myr.

Using the relations derived above, we expect about 15 collisions in 1 million years and about 32 within 10 million years with a single runaway star. We again expect the realistic lifetime of the resulting massive star to be in between these extreme cases. In the case of an atomic cooling halo, we thus conclude that a more considerable enhancement of the mass is possible as a result of stellar collisions.

Also here, the reported values correspond to a mean, and there can be deviations to lower and higher collision fractions. We however expect the number of collisions to remain within the same order of magnitude. 


\section{Discussion and conclusions}

In this paper, we have provided a detailed parameter study showing how the number of collisions and the growth of the mass of the most massive object depends on the properties of the stellar cluster, particularly the number of stars and the stellar radii. We further quantified their time evolution, providing the time when most collisions occur, duration of the peak collision period, and overall normalization via a Gaussian fit. We provided fits and scaling relations on the general results that we found, concerning the characteristic time delay until the maximum of collisions occurs, duration of the period of collisions, and normalization that determines the number of collisions during that period. We applied our results both to the formation of a standard Pop. III star cluster, finding a moderate enhancement of the mass of the most massive star by a factor of a few and to a massive Pop. III cluster in a larger atomic cooling halo, finding a potential enhancement by a factor $15-32$.

Assuming a typical protostellar mass of $20 M_{\odot}$, this would as a result imply resulting black hole masses of up to $600 M_{\odot}$, which are compatible with simulation results by Katz et al. (2015) and Sakurai et al. (2017). We do however note that especially in the massive atomic cooling halos, the properties of the Pop. III clusters are not very well known, and it may be conceivable that a more massive object can form depending on mass and compactness of the clusters. Taking the results obtained here as a conservative estimate, as we neglected the gas-phase processes in this investigation, the resulting black hole masses would correspond to about $0.1-10 \%$ of black hole masses formed via direct collapse (Koushiappas et al. 2004; Lodato \& Natarajan 2007; Latif et al. 2013b; Schleicher et al. 2013; Ferrara et al. 2014).

We find both results potentially relevant. The rather modest increase of the mass of the most massive star in standard Pop. III clusters could, on the one hand, be relevant in cases in which the mass then falls into the range of 130-260 $M_{\odot}$ where pair-instability supernovae are expected to occur (Heger \& Woosley 2002). On the other hand, as no pair-instability abundance patterns have so far been found in extremely metal poor stars (Frebel \& Norris 2015), such a small factor may also explain why pair-instability supernovae has not generally occurred or why such events have been too rare to be found. While it is likely too early to draw definite conclusions, we expect that ongoing efforts through stellar archeology and the upcoming $\mathrm{JWST}^{2}$ will shed light on this issue.

In massive Pop. III clusters, our finding that the mass of the most massive object increases can be expected to be highly relevant. This is particular because the formation of massive seeds via direct collapse still seems highly challenging, requiring both very low metallicities below $10^{-5} Z_{\odot}$ (Omukai et al. 2008; Latif et al. 2016) and very strong radiation backgrounds to dissociate the molecular hydrogen (Latif et al. 2015). Such a scenario may perhaps work as a rare event (e.g., Habouzit et al. 2016). A more recent theory suggests that the existence of mirror dark matter, that is, photons and neutrinos with the same characteristics as in the standard model of particles but interacting only via gravity, could explain the formation of $10^{4}-10^{5} M_{\odot}$ black holes at very high redshift, thereby becoming SMBHs at redshift 7 wthout the need to accrete at super-Eddington rates (D'Amico et al. 2018). If collisions are taken into account, however, the formation of massive objects may become feasible under a broader range of circumstances.

\footnotetext{
2 Webpage JWST: https://www. jwst.nasa.gov/
}

Even if fragmentation occurs, it is very likely that a relevant fraction of the mass nevertheless ends up within the most massive object, as we showed here. In these calculations, we did not yet take into account dissipative effects such as dynamical friction, or the effects resulting from ongoing accretion onto the stars. We therefore expect that particularly within the actively accreting clusters, the number of collisions may be further enhanced, even though this could be partly balanced from the effect of having a more realistic IMF, which could enhance the stellar ejections. While it is clear that further investigations will be required, the present study shows clearly the prospect of forming massive objects via stellar collisions.

Acknowledgements. BR thanks Sverre Aarseth for his help with the code NBODY6. BR also thanks A. Alarcon Jara and D.R. Matus Carrillo for useful discussion during the realization of this work. RSK thanks B. Agarwal, L. Haemmerlé, A. Heger, D. Whalen, and T. Woods for stimulating discussions about massive and supermassive Pop. III stars. DRGS and BR acknowledge funding through Fondecyt regular (project code 1161247). BR thanks Conicyt for financial support (CONICYT-PFCHA/MagísterNacional/2017-22171385). DRGS and MF acknowledge funding through the "Concurso Proyectos Internacionales de Investigación, Convocatoria 2015" (project code PII20150171) and the BASAL Centro de Astrofísica y Tecnologías Afines (CATA) PFB06/2007. DRGS further is grateful for funding via ALMA-Conicyt (project code 31160001), Quimal (project number QUIMAL170001), and Anillo (project number ACT172033). RSK acknowledges financial support from the Deutsche Forschungsgemeinschaft via SFB 881, "The Milky Way System" (subprojects B1, B2, and B8) and SPP 1573, "Physics of the Interstellar Medium". He also appreciates support from the European Research Council via the ERC Advanced Grant "STARLIGHT: Formation of the First Stars" (project number 339177). TB acknowledges support from Fundação para a Ciência e a Tecnologia (grant SFRH/BPD/122325/2016), and support from Center for Research \& Development in Mathematics and Applications (CIDMA) (strategic project UID/MAT/04106/2013), and from ENGAGE SKA, POCI-01-0145FEDER-022217, funded by COMPETE 2020 and FCT, Portugal.

\section{References}

Aarseth, S. J. 2000, in The Chaotic Universe, eds. V. G. Gurzadyan, \& R. Ruffini, 286

Abel, T., Bryan, G. L., \& Norman, M. L. 2002, Science, 295, 93

Ahmad, A., \& Cohen, L. 1973, J. Comput. Phys., 12, 389

Baumgardt, H., \& Klessen, R. S. 2011, MNRAS, 413, 1810

Bodenheimer, P., \& Sweigart, A. 1968, ApJ, 152, 515

Bonnell, I. A., Bate, M. R., \& Zinnecker, H. 1998, MNRAS, 298, 93

Bovino, S., Grassi, T., Schleicher, D. R. G., \& Banerjee, R. 2016, ApJ, 832, 154

Bromm, V., \& Loeb, A. 2003, ApJ, 596, 34

Clark, P. C., Glover, S. C. O., Klessen, R. S., \& Bromm, V. 2011a, ApJ, 727, 110

Clark, P. C., Glover, S. C. O., Smith, R. J., et al. 2011b, Science, 331, 1040

Clarke, C. J., \& Bonnell, I. A. 2008, MNRAS, 388, 1171

D’Amico, G., Panci, P., Lupi, A., Bovino, S., \& Silk, J. 2018, MNRAS, 473, 328 Devecchi, B., Volonteri, M., Colpi, M., \& Haardt, F. 2010, MNRAS, 409, 1057

Devecchi, B., Volonteri, M., Rossi, E. M., Colpi, M., \& Portegies Zwart, S. 2012, MNRAS, 421, 1465

Dopcke, G., Glover, S. C. O., Clark, P. C., \& Klessen, R. S. 2011, ApJ, 729, L3 Dopcke, G., Glover, S. C. O., Clark, P. C., \& Klessen, R. S. 2013, ApJ, 766, 103 Ferrara, A., Salvadori, S., Yue, B., \& Schleicher, D. 2014, MNRAS, 443, 2410 Frebel, A., \& Norris, J. E. 2015, ARA\&A, 53, 631

Fujii, M. S., \& Portegies Zwart, S. 2013, MNRAS, 430, 1018

Greif, T. H., Springel, V., White, S. D. M., et al. 2011, ApJ, 737, 75

Greif, T. H., Bromm, V., Clark, P. C., et al. 2012, MNRAS, 424, 399

Habouzit, M., Volonteri, M., Latif, M., Dubois, Y., \& Peirani, S. 2016, MNRAS, 463,529

Haemmerlé, L., Eggenberger, P., Meynet, G., Maeder, A., \& Charbonnel, C. 2016, A\&A, 585, A65

Haemmerlé, L., Woods, T. E., Klessen, R. S., Heger, A., \& Whalen, D. J. 2018, MNRAS, 474, 2757

Hartwig, T., Volonteri, M., Bromm, V., et al. 2016, MNRAS, 460, L74

Heger, A., \& Woosley, S. E. 2002, ApJ, 567, 532

Hosokawa, T., Omukai, K., \& Yorke, H. W. 2012, ApJ, 756, 93

Hosokawa, T., Yorke, H. W., Inayoshi, K., Omukai, K., \& Yoshida, N. 2013, ApJ, 778,178

Hurley, J. R., Pols, O. R., \& Tout, C. A. 2000, MNRAS, 315, 543 
Katz, H., Sijacki, D., \& Haehnelt, M. G. 2015, MNRAS, 451, 2352

Keto, E., \& Klaassen, P. 2008, ApJ, 678, L109

Klessen, R. S., Glover, S. C. O., \& Clark, P. C. 2012, MNRAS, 421, 3217

Koushiappas, S. M., Bullock, J. S., \& Dekel, A. 2004, MNRAS, 354, 292

Krumholz, M. R., Klein, R. I., McKee, C. F., Offner, S. S. R., \& Cunningham, A. J. 2009, Science, 323, 754

Kustaanheimo, P., \& Stiefel, E. 1965, J. Reine Angew. Math., 218, 204

Larson, R. B. 1969, MNRAS, 145, 271

Latif, M. A., \& Schleicher, D. R. G. 2015, MNRAS, 449, 77

Latif, M. A., Schleicher, D. R. G., Schmidt, W., \& Niemeyer, J. 2013a, ApJ, 772, L3

Latif, M. A., Schleicher, D. R. G., Schmidt, W., \& Niemeyer, J. C. 2013b, MNRAS, 436, 2989

Latif, M. A., Schleicher, D. R. G., Bovino, S., Grassi, T., \& Spaans, M. 2014, ApJ, 792, 78

Latif, M. A., Bovino, S., Grassi, T., Schleicher, D. R. G., \& Spaans, M. 2015 MNRAS, 446, 3163

Latif, M. A., Omukai, K., Habouzit, M., Schleicher, D. R. G., \& Volonteri, M. 2016, ApJ, 823, 40

Lodato, G., \& Natarajan, P. 2007, MNRAS, 377, L64

Lupi, A., Colpi, M., Devecchi, B., Galanti, G., \& Volonteri, M. 2014, MNRAS, 442,3616

Maeder, A., \& Meynet, G. 2012, Rev. Mod. Phys., 84, 25

Moeckel, N., \& Clarke, C. J. 2011, MNRAS, 410, 2799

Oh, S., \& Kroupa, P. 2012a, MNRAS, 424, 65

Oh, S., \& Kroupa, P. 2012b, MNRAS, 424, 65

Oh, S., \& Kroupa, P. 2016, A\&A, 590, A107

Oh, S., Kroupa, P., \& Pflamm-Altenburg, J. 2015, ApJ, 805, 92
Omukai, K., \& Palla, F. 2001, ApJ, 561, L55

Omukai, K., \& Palla, F. 2003, ApJ, 589, 677

Omukai, K., Tsuribe, T., Schneider, R., \& Ferrara, A. 2005, ApJ, 626, 627

Omukai, K., Schneider, R., \& Haiman, Z. 2008, ApJ, 686, 801

Palla, F., \& Stahler, S. W. 1993, ApJ, 418, 414

Peters, T., Banerjee, R., Klessen, R. S., et al. 2010a, ApJ, 711, 1017

Peters, T., Klessen, R. S., Mac Low, M.-M., \& Banerjee, R. 2010b, ApJ, 725, 134

Peters, T., Banerjee, R., Klessen, R. S., \& Mac Low, M.-M. 2011, ApJ, 729, 72

Pflamm-Altenburg, J., \& Kroupa, P. 2010, MNRAS, 404, 1564

Plummer, H. C. 1911, MNRAS, 71, 460

Rees, M. J. 1984, ARA\&A, 22, 471

Sakurai, Y., Yoshida, N., Fujii, M. S., \& Hirano, S. 2017, MNRAS, 472, 1677

Schaerer, D. 2002, A\&A, 382, 28

Schleicher, D. R. G., Palla, F., Ferrara, A., Galli, D., \& Latif, M. 2013, A\&A, 558, A59

Schneider, R., Ferrara, A., Salvaterra, R., Omukai, K., \& Bromm, V. 2003, Nature, 422, 869

Schneider, R., Omukai, K., Inoue, A. K., \& Ferrara, A. 2006, MNRAS, 369, 1437

Schneider, R., Omukai, K., Limongi, M., et al. 2012, MNRAS, 423, L60

Shu, F. H. 1977, ApJ, 214, 488

Smith, R. J., Glover, S. C. O., Clark, P. C., Greif, T., \& Klessen, R. S. 2011, MNRAS, 414, 3633

Smith, R. J., Hosokawa, T., Omukai, K., Glover, S. C. O., \& Klessen, R. S. 2012, MNRAS, 424, 457

Stahler, S. W., Palla, F., \& Salpeter, E. E. 1986, ApJ, 302, 590

Woods, T. E., Heger, A., Whalen, D. J., Haemmerlé, L., \& Klessen, R. S. 2017, ApJ, 842, L6

Yorke, H. W., \& Sonnhalter, C. 2002, ApJ, 569, 846 